\title{
Multi-channel SNSPD system with high detection efficiency at telecommunication wavelength
}

\author{
Shigehito Miki, ${ }^{1, *}$ Taro Yamashita, ${ }^{1}$ Mikio Fujiwara, ${ }^{2}$ Masahide Sasaki, ${ }^{2}$ and Zhen Wang $^{1}$ \\ ${ }^{1}$ Kansai Advanced Research Center, National Institute of Information and Communications \\ Technology, 588-2, Iwaoka, Iwaoka-cho, Nishi-ku, Kobe, Hyogo 651-2492, Japan \\ ${ }^{2}$ National Institute of Information and Communications Technology, 4-2-1 Nukui-Kitamachi, \\ Koganei, Tokyo 184-8795, Japan \\ *Corresponding author: s-miki@nict.go.jp
}

We developed a four-channel superconducting nanowire single-photon detector system based on a Gifford-McMahon cryocooler. All channels showed a system detection efficiency (at a $100 \mathrm{~Hz}$ dark-count rate) higher than $16 \%$ at $1550 \mathrm{~nm}$ wavelength, and the best channel showed a system DE of $21 \%$ and $30 \%$ at $1550 \mathrm{~nm}$ and $1310 \mathrm{~nm}$ wavelength, respectively.

OCIS codes: $030.5260,040.3060,040.5160,270.5570$. 
In recent times, multichannel superconducting nanowire single-photon detector (SNSPD [1]) systems based on closed-cycle cryocoolers have been recognized as promising instruments in the field of optical quantum information technology; this is because SNSPDs deliver a good performance and are capable of continuous and stable operation without any liquid cryogen. At present, the type of SNSPDs mostly used in the multichannel systems are single-layer nanowire devices. They typically showed a practical system detection efficiency (DE) of 1-3\% at $1550 \mathrm{~nm}$ wavelength, low dark-count rate (DCR) of 10-100 Hz, and excellent timing jitter of 30-100 ps $[2,3]$. Although they have been successfully employed in quantum key distribution (QKD) $[2,4-$ 6], further improvement in the system performance, especially in the system DE, is desired.

An effective method of improving the system DE is enhancement of the photoabsorption coefficient by integrating an optical cavity structure with the SNSPD device (OC-SNSPD)[7]. Moreover, efficient optical coupling to the meander nanowire area simultaneously is crucial, and a primary concern is how to implement the OC-SNSPDs in practical multichannel system. Although the successful implementation of a single OC-SNSPD in a practical closed-cycle cryocooler systems has been reported $[8,9]$, the development of multichannel systems has not yet been reported. Since the BB84 [10] protocol, which is currently a most matured protocol with complete security certification, requires four channels at the receiver side [5], it is necessary to have four channels in the system for use in these applications. In this letter, we report the development of a practical four-channel OC-SNSPD system with higher system DE than $16 \%$ at a wavelength of $1550 \mathrm{~nm}$. We present a newly developed compact fiber-coupled packaging technique using lenses and describe their system DE and DCR performances.

The devices used in this study were patterned onto 4-nm-thick $\mathrm{NbN}$ thin films on $\mathrm{MgO}$ substrates [11]. We fabricated 80 -nm-wide $\mathrm{NbN}$ meander nanowires covering an area of $15 \times 15$ 
$\mu \mathrm{m}^{2}$ with a filling factor of $62.5 \%$. The superconducting critical temperature $\mathrm{T}_{c}$ and critical current density $\mathrm{J}_{\mathrm{c}}$ of nanowires were $10.2-10.5 \mathrm{~K}$ and $4-7 \times 10^{10} \mathrm{~A} / \mathrm{m}^{2}$, respectively. An optical cavity structure consisting of a 100-nm-thick Au mirror and a 250-nm-thick SiO cavity were covered on the meander nanowire area. The thicknesses of $\mathrm{Au}$ mirror and $\mathrm{SiO}$ cavity were designed for wavelengths of $1300-1600 \mathrm{~nm}$.

Figure 1 (a) shows the schematic layout of the fiber-coupled packaging for OC-SNSPDs. This compact fiber-coupled packaging technique was modified from the one used for single layer SNSPD [2,3], which is simple and has high reliability. A fiber ferrule was fixed to the fiberholding block in advance by using an adhesive so that the distance from the exit end to the rear surface of the OC-SNSPD chip was $20 \mu \mathrm{m}$ at low temperature. OC-SNSPD chips were mounted on chip-mounting blocks which had a through hole at the center of the chip-mounting area. An MU-type fiber ferrule was inserted through this hole from the rear. The fiber-holding block was joined to the chip-mounting block from the rear, and the two blocks were accurately aligned so that the incident light spot illuminated the center of the meander area. The dimensions of the packaging blocks which could be used for OC-SNSPDs are almost of the same size $(15 \mathrm{~mm}$ (length) $\times 15 \mathrm{~mm}($ width $) \times 10 \mathrm{~mm}$ (thickness) $)$ as those used for single-layer SNSPD chips, as shown in fig.1 (b). Hence, OC-SNSPD packaging blocks were installed in the multichannel Gifford-McMahon (GM) cryocooler system without any modification, which can simultaneously cool six SNSPD packages to $2.9 \mathrm{~K}$ with a thermal fluctuation range of $\pm 10 \mathrm{mK}$ [3].

To achieve efficient optical coupling, light beam waist on the meander nanowire area must be smaller than the size of the nanowire area. Since the OC-SNSPDs have to be illuminated from the rear side through the substrate, small-gradient index (GRIN) lenses were used to reduce the beam waist at a distant from exit-end. In order to embed lenses into the compact packages, 
GRIN lenses with a diameter of $125 \mu \mathrm{m}$, which is equal to the clad diameter of a single-mode (SM) optical fiber, are directly fusion spliced to the end of the optical fiber. Since the fiberspliced lenses were inserted into the MU fiber ferrule, the shape of the end of fiber did not change at all from that without lenses, as shown in Fig.1 (c). The numerical aperture and length of the two lenses are chosen so that the focal length is equal to the appropriate distance in the packaging and the beam waist becomes as small as possible. As a result, the beam waist $\left(2 \omega_{0}\right)$ was estimated to be $8-10 \mu \mathrm{m}$ on the meander nanowire area, when the distance between the exitend and the substrate is $20 \mu \mathrm{m}$ and the thickness of the $\mathrm{MgO}$ substrate is $400 \mu \mathrm{m}$. This beam waist is sufficiently small to allow efficient optical coupling with the meander nanowire area of $15 \times 15 \mu \mathrm{m}^{2}$.

To verify the effectiveness of the fiber-spliced GRIN lenses, we measured the system DE versus the DCR of the OC-SNSPD device, with and without the lenses, as shown in Figure 2. Here, system DE is defined as the ratio of the output count rate and the input photon flux to the system. The system DE (at a DCR of $100 \mathrm{~Hz}$ ) of a device without lenses remained to be a small value of $2.8 \%$; this was because the beam waist expanded on the meander nanowire area, thereby resulting in poor optical coupling. With focusing by GRIN lenses, system DE was much improved to be $21 \%$. Figure 3(a) shows system DE versus DCR and Fig. 3(b) shows the system DE and DCR versus bias current normalized by superconducting critical current $I_{c}$ at wavelengths of $1310 \mathrm{~nm}$ and $1550 \mathrm{~nm}$. A maximum system DE reached 40\% and 28\%, at 1310 $\mathrm{nm}$ and $1550 \mathrm{~nm}$ wavelength, respectively, at the DCR of several thousand $\mathrm{Hz}$, where the bias current was just below $\mathrm{I}_{\mathrm{c}}\left(\sim 0.99 \mathrm{I}_{\mathrm{c}}\right)$. This implies the optical cavity also worked efficiently at $1310 \mathrm{~nm}$ wavelength and the device DE at $1310 \mathrm{~nm}$ became higher than that at $1550 \mathrm{~nm}$. 
By using compactly packaged OC-SNSPDs, we built up a four-channel SNSPD system. Figure 4 shows the system DE versus DCR of the four channel OC-SNSPDs at a wavelength of $1550 \mathrm{~nm}$. Needless to say, all the channels can operate simultaneously without any time gating, and hence, our OC-SNSPD can be used in QKD protocols and other quantum optical applications. The system DE of all the channels show similar dependencies on the DCR and exceed $16 \%$ and $20 \%$ at DCRs of $100 \mathrm{~Hz}$ and $2000 \mathrm{~Hz}$ respectively, which are significantly higher than those of standard devices [4]. Although the system DE varies from channel to channel, we believe values of all the channels can exceed those achieved in the present study by improving the yield of nanowire uniformity. The performance of the packaged OC-SNSPDs do not show any significant change during several thermal cycles. These results indicate that our packaging technique exhibits a high stability against thermal cycling.

As a future study, an effective optical coupling to smaller devices should be considered because miniaturization of device size are certainly effective for further improvement of both device DE and response speed $[12,13]$. It should be noted that this will be possible by utilizing a combination of fiber-spliced GRIN lenses and substrate thickness-reduction technique [9]. For example, if the thickness of the $\mathrm{MgO}$ substrate is reduced to $50 \mu \mathrm{m}$ and the GRIN lenses are optimally designed, the beam waist can be reduced to $4-5 \mu \mathrm{m}$, thus making it possible to achieve efficient optical coupling to smaller devices.

In conclusion, we have developed a four-channel SNSPD system using OC-SNSPD devices. To achieve efficient optical coupling in the multichannel system, a compact fibercoupled packaging technique that employ GRIN lenses has been developed. The beam waist on the meander nanowire area has been successfully reduced for achieving effective optical coupling to devices with an area of $15 \times 15 \mu \mathrm{m}^{2}$. All the channels showed a system DE higher 
than $16 \%$, and best device showed $21 \%$ at a DCR of $100 \mathrm{~Hz}$ and wavelength of $1550 \mathrm{~nm}$. These DE values are significantly higher than those of standard multi-channel SNSPD systems, and definitely make a great impact to the QKD and various applications. 
(a)
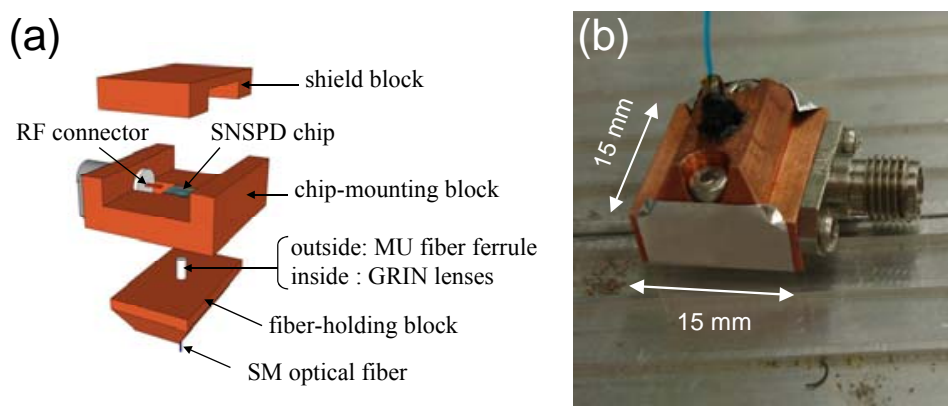

(c)

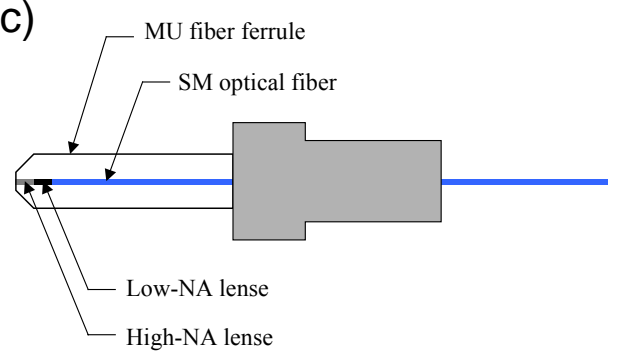

Fig. 1. (a) Schematic layout and (b) photograph of fiber-coupled packaging for OC-SNSPDs (c) configuration of GRIN lenses connected to optical fiber.

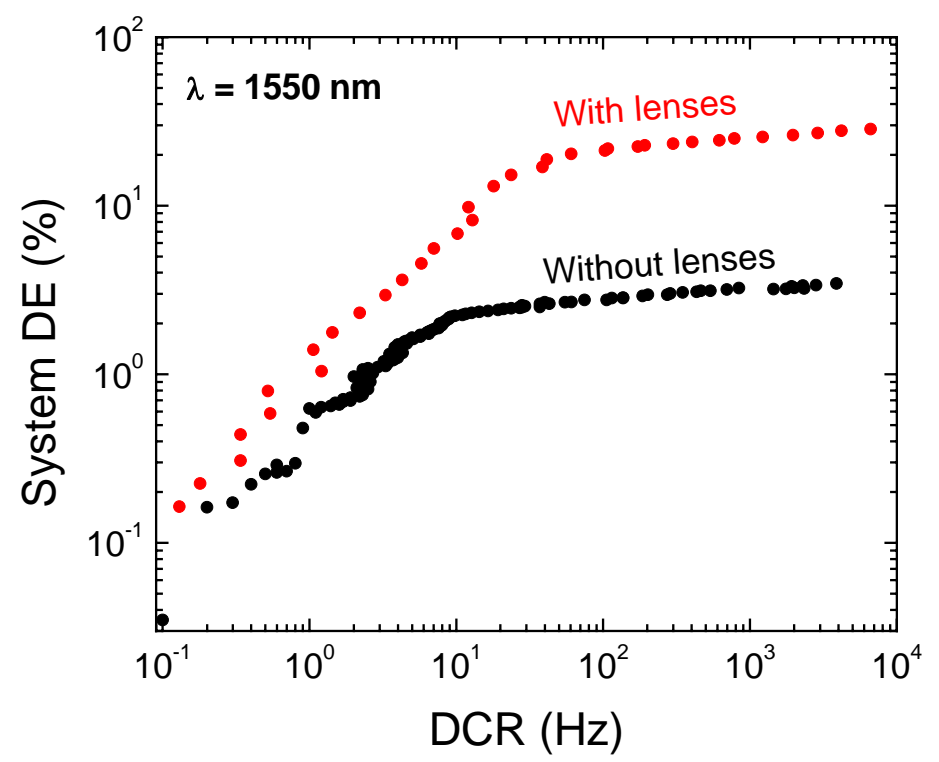

Fig.2. System DE versus DCR of OC-SNSPD device packaged with and without GRIN lenses.

The System DE (at a $100 \mathrm{~Hz}$ DCR) reached a level of $21 \%$ by using GRIN lenses 


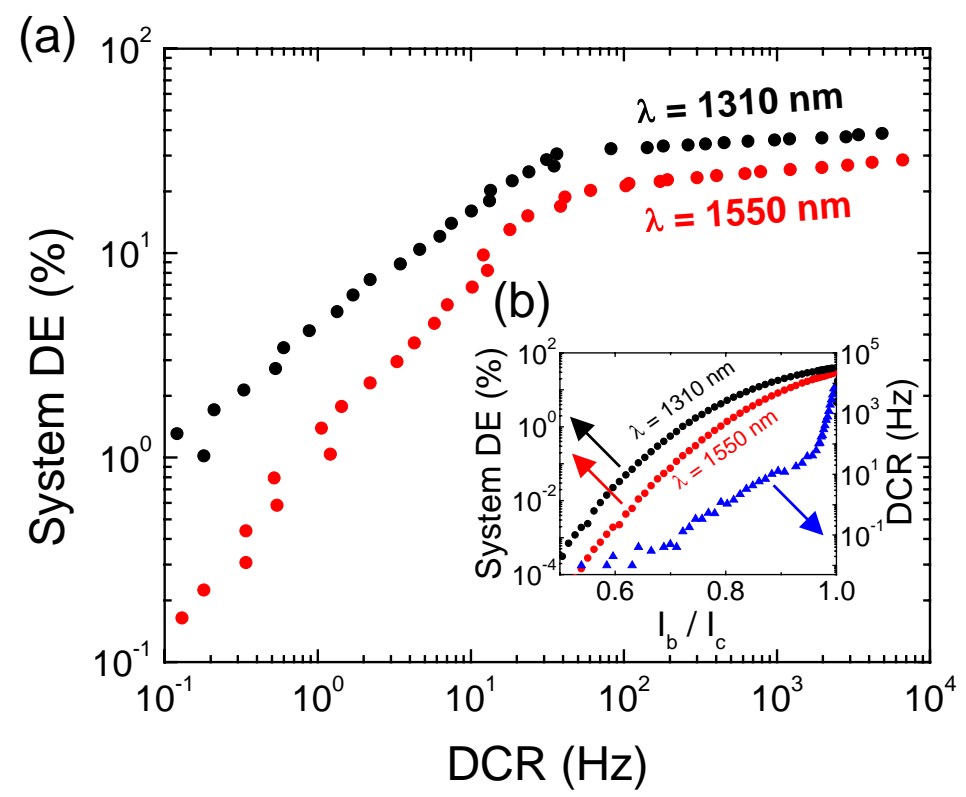

Fig.3. The best performance of our OC-SNSPD device. (a)System DE versus DCR and (b) system DE and DCR versus bias current of OC-SNSPD device at $1310 \mathrm{~nm}$ and $1550 \mathrm{~nm}$ wavelength.

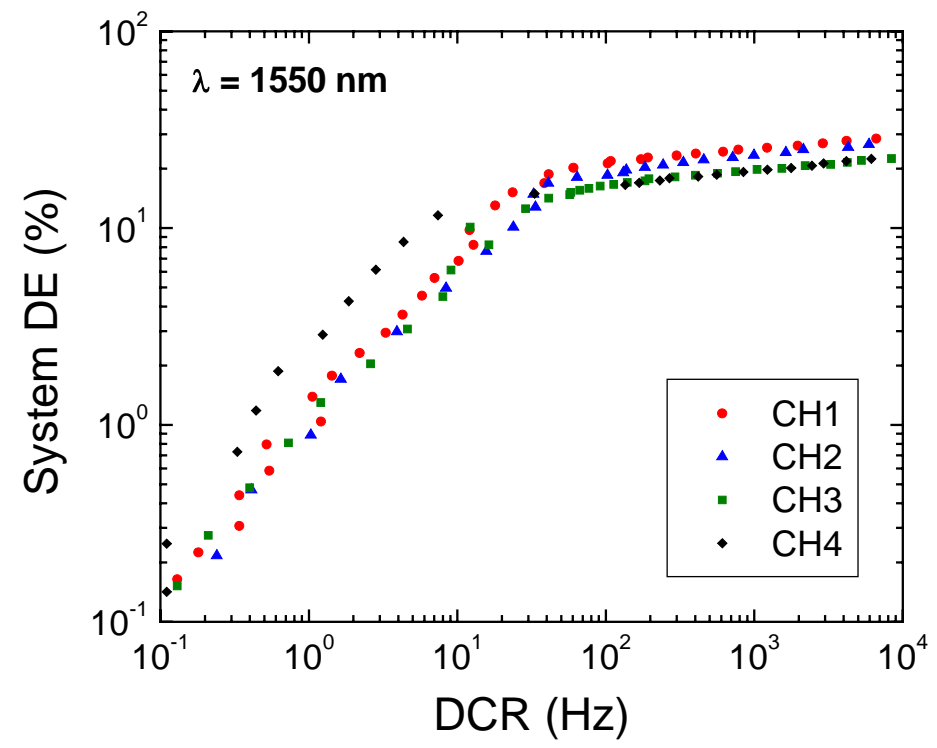

Fig.4. System DE versus DCR of four-channel OC-SNSPDs at a wavelength of $1550 \mathrm{~nm}$. 


\section{References}

1. G. Gol'tsman, O. Okunev, G. Chulkova, A. Lipatov, A. Semenov, K. Smirnov, B. Voronov, A. Dzardanov, C. Williams, and R. Sobolewski, “Picosecond superconducting single photon detector," Appl. Phys. Lett. 79, 705-707 (2001).

2. R. H. Hadfield, M. J. Stevens, S. S. Gruber, A. J. Miller, R. E. Schwall, R. P. Mirin, and S. W. Nam, "Single photon source characterization with a superconducting single photon detector," Opt. Express 13, 10846-10853 (2005).

3. S. Miki, M. Fujiwara, M. Sasaki, and Z. Wang, "Development of SNSPD System with Gifford-McMahon Cryocooler,” IEEE. Trans. Appl. Supercond.19, 332-335 (2009).

4. H. Takesue, S. Nam, Q. Zhang, R. H. Hadfield, and Y. Yamamoto, "Quantum key distribution over a 40-dB channel loss using superconducting single-photon detectors," Nat. Photonics 1, 343-348 (2007).

5. A. Tanaka, M. Fujiwara, S. W. Nam, Y. Nambu, S. Takahashi, W. Maeda, K. Yoshino, S. Miki, B. Baek, Z. Wang, A. Tajima, M. Sasaki, and A. Tomita, "Ultra fast quantum key distribution over a $97 \mathrm{~km}$ installed telecom fiber with wavelength division multiplexing clock synchronization,” Opt. Express 16, 11354-11360 (2008).

6. T. Honjo, S. W. Nam, H. Takesue, Q. Zhang, H. Kamada1, Y. Nishida, O. Tadanaga, M. Asobe, B. Baek, R. Hadfield, S. Miki, M. Fujiwara, M. Sasaki, Z. Wang, K. Inoue, and Y. Yamamoto, “ Long-distance entanglement-based quantum key distribution over optical fiber,” Opt. Express 16, 19118-19126 (2008).

7. K. M. Rosfjord, J. K. W. Yang, E. A. Dauler, A. J. Kerman, V. Anant, B. M. Boronov, G. N. Gol'tsman, and K. K. Berggren, "Nanowire single photon detector with an integrated optical cavity and anti-reflection coating," Opt. Express 14, 527-534 (2006). 
8. X. Hu, T. Zhong, J. E. White, E. A. Dauler, F. Najafi, C. H. Herder, F. N. C. Wong, and K. Berggren, " Fiber-coupled nanowire photon counter at $1550 \mathrm{~nm}$ with $24 \%$ system detection efficiency," Opt. Lett. 34, 3607-3609 (2009).

9. S. Miki, M. Takeda, M. Fujiwara, M. Sasaki, and Z. Wang, " Compactly packaged superconducting nanowire single-photon detector with an optical cavity for multichannel system," Opt. Express 17, 23557-23564 (2009).

10. C. H. Bennett and G. Brassard, "Quantum Cryptography: Public Key Distribution and Coin Tossing," in Proceedings of the IEEE International Conference on Computers, Systems and Signal Processing (IEEE, New York, 1984), pp. 175-179

11. S. Miki, M. Fujiwara, M. Sasaki, and Z. Wang, "NbN Superconducting Single-photon Detectors Prepared on Single-crystal MgO Substrates," IEEE Trans. Appl. Supercond. 17, $285-288$ (2007).

12. A. J. Kerman, E. A. Dauler, W. E. Keicher, J. K. W. Yang, K. K. Berggren, G. N. Gol'tsman, and B. M. Voronov, " Kinetic-inductance-limited reset time of superconducting nanowire photon counters," Appl. Phys. Lett. 88, 111116 (2006).

13. A. J. Kerman, E. A. Dauler, J. K. W. Yang, K. M. Rosfjord, K. K. Berggren, G. Gol'tsman, and B. Voronov, " Constriction-limited detection efficiency of superconducting nanowire single-photon detectors," Appl. Phys. Lett. 90, 101110 (2007). 